\title{
In Vitro Release Testing Methodology and Variability with the Vertical Diffusion Cell (VDC)
}

Ryan R. Klein ${ }^{1,2, *}$, Jenna L. Heckart ${ }^{1,3}$, and Kailas D. Thakker ${ }^{1}$

${ }^{1}$ Tergus Pharma, Durham NC, USA

${ }^{2}$ Alliance Pharma, Malvern PA, USA

${ }^{3}$ Bioagilytix, Durham NC, USA

\section{ABSTRACT}

In vitro release test (IVRT) using the vertical diffusion cell (VDC) is a well-established method to evaluate the performance of a topical dosage form and to better understand the physicochemical characteristics of the product. The VDC is the most frequently used apparatus to measure drug release from semisolid products and has been in use for more than fifty years; however, the technique is often hampered by high variability and inadequate reproducibility. Various method parameters influence drug release and the associated variation of the release profiles. The purpose of this manuscript is to examine the critical parameters of the VDC apparatus and the methods that are developed using it, and to highlight potential sources of variability that must be well understood to develop, validate, and implement highly effective VDC methods.

KEYWORDS: In vitro release rate, vertical diffusion cell, VDC, variability

\section{INTRODUCTION}

$\mathrm{n}$ vitro release testing (IVRT) as a tool to support topical product development has been around for approximately 50 years. Early studies were performed to understand the release characteristics of traditional steroid creams and ointments that were available at the time; Shah et. al. and several others carried out many release rate determinations of hydrocortisone and betamethasone dipropionate semisolid products $(1,2)$. These early years also included the introduction of the vertical diffusion cell (VDC) and coining of the term 'Franz' diffusion cell (3).

The VDC is the most frequently used apparatus to develop and validate IVRT methods. There are other acceptable systems, such as the immersion cell and USP Apparatus 4, but these systems are beyond the scope of this article. The VDC is usually made of borosilicate glass and is comprised of two compartments: a donor chamber that holds the formulation during the course of the experiment, and a receptor chamber that contains an appropriately selected receptor fluid and serves as the sampling site during the experiment. The receptor chamber is designed to maintain a constant temperature via either a water jacket or dry heating source. Between the donor and receptor chamber sits an appropriately selected synthetic, inert membrane, which acts as a holding surface for the formulation and serves as a demarcation between the two chambers. The membrane is intended to keep the drug product and receptor fluid completely separated. USP Chapter <1724> describes the VDC in greater detail and recommends specific dimensions for the apparatus based on input from leaders in industry, academia, and regulatory agencies (4). Various dimensions are described with orifice diameter, receptor volume, and donor chamber size being the most critical to understand to keep variability as low as possible. During the last 20 years, various publications have reviewed various aspects and applications of IVRT as a tool to support topical product development, from scale-up and post-approval changes for non-sterile semisolid dosage forms (SUPAC-SS) to the more recently proposed topical drug classification system (TCS) (5-9).

In 1997, the first regulatory document regarding the use of IVRT was published to support SUPAC-SS. This guidance from the agency allowed the use of IVRT in lieu of clinical trials for certain levels of changes in commercial products. Several levels of changes, including changes in manufacturing site and scale, changes in manufacturing process and equipment, and certain changes to the components and composition of the drug product (e.g., excipient grade or supplier and amounts of individual excipients) allow the use of IVRT as a tool to demonstrate equivalency between pre- and post-manufactured batches in lieu of clinical trials (10). As a result of this 
initial regulatory publication, the demand for developing IVRT methods across the pharmaceutical industry grew substantially and started to become essential to core development programs for topical drug products. Regulatory expectations for the use of $Q b D$ as part of topical product development also increased, creating the needfor additional tools to optimize product performance. Thus, the application of IVRT to understand changes in release mechanisms with changes in composition and process parameters emerged and allowed better insight into the development of high quality topical products.

Whether developing a new drug product or a generic equivalent, developing an IVRT method in parallel with the product allows optimization of the formulation and manufacturing process at various stages of development. The release properties of the active ingredient from the formulation provide insight into the microstructure of the product, particularly during long-term stability studies, and therefore allow optimization of the physical characteristics of the formulation during product development. The IVRT method can also provide support for sameness of novel drug products between stages of clinical development by establishing sameness as minor formulation changes are implemented during the course of clinical assessment. Each of these applications is critical to reduce risk throughout the development program and justifies implementation of this performance test very early in the overall product development strategy.

Over the last 20 years, regulatory approval of generic medicines has increased significantly; however, regulatory approvals for locally acting topical generic drugs have lagged behind. For locally acting drugs, measuring drug concentrations at the site of action is often not feasible, and plasma concentrations are rarely a good indicator of bioequivalence (BE). As a result, clinical endpoint studies are frequently necessary to establish $\mathrm{BE}$, and are a substantial barrier to approval of branded and unbranded generic drug products that exert their effect locally at the site of administration. To overcome the barriers to developing topical generic products, the US Food and Drug Administration (FDA) has implemented new strategies. For example, when a generic product meets criteria for qualitative (Q1) and quantitative (Q2) similarity, IVRT, along with some physicochemical and/or pharmacodynamic testing, can be used to demonstrate microstructure (Q3) similarity as an alternative to conducting clinical trials. Several bioavailability (BA) and $\mathrm{BE}$ guidance documents have been published since 2005. While the first guidance published for acyclovir ointment was very short and general, the more recent guidance documents on acyclovir cream, dapsone gel, and ivermectin cream are very specific and detailed, and outline requirements for development and validation of the high-performance liquid chromatography (HPLC) and IVRT methods, as well as for the pivotal study comparison of the generic to the reference product (11-14).

\section{METHOD VALIDATION}

Once a suitable IVRT method has been developed for a specific product, it should be fully validated at the appropriate time during product development. Various publications over the last 20 years have highlighted approaches to validated release methods using VDCs (15-18). In the aforementioned FDA guidance documents, the agency has recently described in detail the regulatory expectations for method validations, starting with the VDC (12). The apparatus should be appropriately qualified by confirming the surface area, diffusion cell volume, temperature control, and stirring rate. Appropriate laboratory environmental controls should also be monitored and maintained while conducting the entire set of studies. The validation should include proper assessment of the following method attributes: 1) drug substance solubility and stability in the receptor fluid; 2) drug substance binding to the membrane; 3 ) intra-run and inter-run precision of release rates; 4 ) linearity of release rate; 5) recovery, mass balance, and dose depletion; 6) discrimination sensitivity; 7) discrimination selectivity; 8) discrimination specificity; 9) supplemental discrimination selectivity using altered formulations; and 10) robustness with respect to the most critical method parameters (e.g., dose volume, receptor fluid composition and/or $\mathrm{pH}$, stirring rate, temperature). Specific descriptions of each of the aforementioned attributes can be found in the FDA guidance document for acyclovir cream (12). Validation of the HPLC method is recommended to be consistent with either the current FDA guidance on Bioanalytical Method Validation and/or the ICH Harmonized Tripartite Guideline on Validation of Analytical Procedures Q2(R1) $(19,20)$. Although the IVRT sample matrix is not as complex as some biological matrices, the bioanalytical method validation principles are frequently expected, presumably because IVRT is used to support a biowaiver for a clinical study (4).

\section{VDC METHOD PARAMETERS}

VDC methods have several parameters that must be tightly controlled to achieve optimum, acceptable performance. The primary method parameters include the temperature of the receptor fluid, diffusion cell dimensions (e.g., orifice diameter, size of the donor chamber, and receptor volume), sampling times, membrane, receptor fluid, stirring rate, amount of 
product to apply to the donor chamber, and application method. During development of the VDC method, each of these parameters should be appropriately selected and justified with experimental data. Careful selection of each parameter leads to developed methods that are precise, reproducible, discriminatory for various alterations in the dosage form, and suitable for validation. On the other hand, inadequately chosen parameters can lead to methods with high intra- and inter-experiment variability that makes it difficult to draw adequate conclusions from the data.

Each of the aforementioned method parameters will be examined in greater detail, followed by a discussion regarding how each these method parameters can contribute to the overall variability in IVRT methods using VDC.

\section{Temperature}

The temperature of the receptor fluid at the membrane surface should be tightly controlled and justified based on the site of application. For most products intended for application to the skin surface, $32{ }^{\circ} \mathrm{C} \pm 1{ }^{\circ} \mathrm{C}$ is suitable; for products intended for rectal or vaginal application, $37^{\circ} \mathrm{C}$ $\pm 1{ }^{\circ} \mathrm{C}$ is more appropriate (4). The temperature should be measured in each diffusion cell prior to application of the drug product to the donor chamber and at the conclusion of the experiment to confirm the temperature was within the appropriate range throughout the experiment.

\section{VDC Dimensions}

Diffusion cells with various dimensions are commercially available from several vendors. USP chapter <1724> describes the dimensions that are generally acceptable. If other dimensions of a VDC are used, they should be appropriately justified. Three dimensions of the diffusion cell should be carefully considered prior to developing a method: the size of the donor chamber, the orifice diameter, and the volume of the receptor chamber. First, the size (i.e., volume capacity) of the donor chamber can impact the ability to maintain the infinite dose assumption during the experiment. If the active ingredient releases quickly into the receptor chamber, and a large percentage of the applied dose is released into the receptor chamber during the experiment, a larger donor chamber may be necessary in combination with an increased application amount to maintain the infinite dose. Second, the orifice diameter can impact the percentage of the applied dose that is released into the receptor chamber during the experiment. The percent released can be minimized by using a smaller orifice diameter. Conversely, the percent released can be increased by using a larger orifice diameter.
Third, the volume of the receptor chamber can be used to increase or decrease the capacity of the receptor fluid to ensure that 'sink' conditions are maintained at the terminal sampling time. The larger the receptor volume, the larger the 'sink' becomes. The optimal dimensions of the diffusion cell will be product-specific and should be appropriately justified using experimental data.

As an example of the impact of VDC design on drug release, Figure 1 presents the release of hydrocortisone from hydrocortisone cream using 70:30 water:ethanol as the receptor fluid and a Tuffryn membrane. Two sets of diffusion cells were used with different dimensions. The larger cells have a receptor chamber with a volume of approximately $12 \mathrm{~mL}$ and surface area of $1.767 \mathrm{~cm}^{2}$, while the small cells have a receptor chamber with a volume of approximately $8 \mathrm{~mL}$ and surface area of $1.0 \mathrm{~cm}^{2}$. The amount of cream applied to the donor chamber was 400 $\mathrm{mg}$ for large cells and $1 \mathrm{~g}$ for small cells. Although the release rates are similar and equivalent, the percent of applied dose released is $12 \%$ for large cells as opposed to only $2.5 \%$ for small cells; the percent released was reduced with a larger application amount and smaller orifice. Although in this case the release rates did not change significantly, different cell dimensions can lead to vastly different release rates for the same formulation and same VDC method.

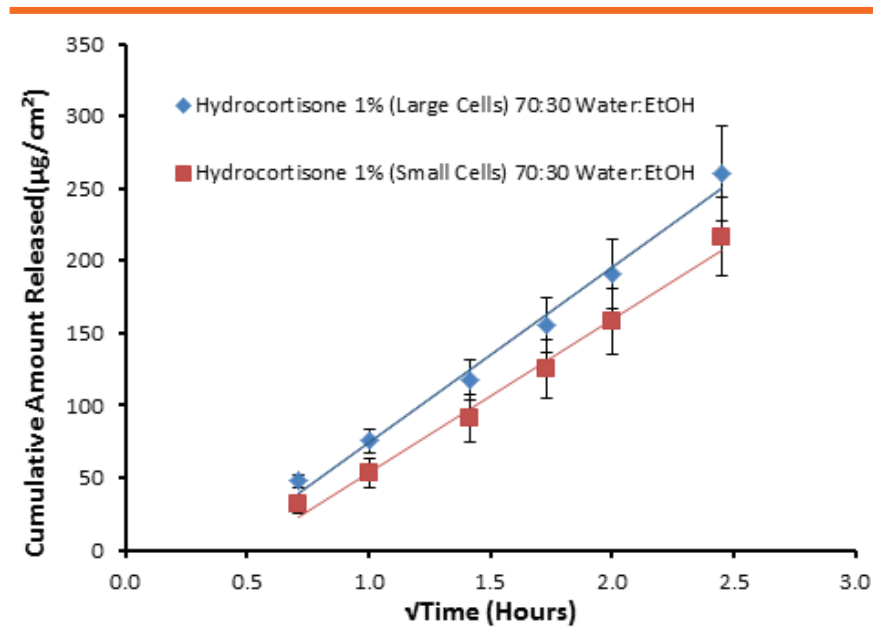

Figure 1. Release of hydrocortisone from a cream formulation using vertical diffusion cells with different dimensions.

\section{Sampling Times and Procedure}

Sampling is generally performed over a 4-6-hour period with a minimum of five samples per diffusion cell $(4$, 10). This duration is experimentally practical, and the identification of a linear region of release can typically be achieved within this time frame when other method parameters are properly selected. The sampling times should be chosen to ensure that the cumulative amount 
of drug released is linear with respect to the square root of time $(4,10)$. When an aliquot of the receptor fluid is withdrawn, the same volume of pre-warmed receptor fluid should be immediately replaced such that the lower surface of the membrane remains in contact with the receptor fluid throughout the experiment. The samples should be pulled from a location within the middle of the body of the diffusion cell that is properly mixed.

\section{Synthetic Membrane}

The synthetic membrane is intended to serve as an inert holding surface for the formulation during the course of the experiment. The membrane will cover the orifice at the intersection between the donor and receptor chambers; the orifice itself, and not the membrane, will define the surface area available for the formulation. There are a variety of commercially available synthetic membranes that can be used successfully during IVRT experiments (e.g. PTFE (polyethylenetertafluoroethylene), PVDF (polyvinylidene fluoride), polycarbonate, polysulfone, polyethersulfone, nylon, and cellulose). Each of these membrane types can be purchased from several vendors with varying diameters and pore sizes.

The synthetic membrane should not be the rate-limiting factor for drug release, bind to the drug substance in the product, or create any interference in the analytical assay. The synthetic membrane should be compatible with the drug product and the selected receptor fluid.

\section{Receptor Fluid}

The receptor fluid is perhaps the most critical parameter to select during development of the IVRT method. The receptor fluid should have high capacity to dissolve the drug substance to ensure that sink conditions are maintained throughout the experiment (i.e., release rate of the active ingredient should not be limited by the drug solubility in the receptor fluid and the solubility of the active ingredient in the receptor is five to 10 times higher than the concentration in the sample taken at the final timepoint). Methods that do not meet the definition of sink conditions should not be used to draw conclusions regarding drug product performance. The chosen solvents should also be compatible with the membrane, as well as the analytical methodology for analysis of the samples. The active ingredient should be sufficiently stable in the receptor fluid, and data should be generated to support stability during the experiment at the appropriate temperature, during the analysis to support re-injection of samples if necessary, and during storage after collection, if desired. In addition, the receptor fluid should provide linear and precise release rates during a single experiment, adequate reproducibility of release rates from experiment to experiment and from scientist to scientist, and sufficient discrimination between intentionally altered formulations as described in the FDA guidance document for acyclovir cream (12).

\section{Stirring Rate}

The stirring rate should be consistent across all diffusion cells. The nominal value of many commercially available diffusion cell systems is $600 \mathrm{rpm}$, although other speeds can be justified provided adequate mixing of the receptor fluid is achieved throughout the experiment.

\section{Amount of Drug Product}

Generally, not less than $300 \mathrm{mg}$ of the drug product is applied to the donor chamber, which is typically sufficient to completely cover the exposed membrane surface $(4,10)$. During the experiment, the release kinetics of the drug substance should be within the requirements of Higuchi's assumptions of an infinite dose to ensure optimal performance of the method (21-24). Generally speaking, no more than $30 \%$ of the applied drug substance should be released into the receptor fluid during the experiment to ensure an infinite dose is maintained in the donor chamber; therefore, amounts larger than 300 $\mathrm{mg}$ are justified to ensure an infinite dose is maintained throughout the experiment. Amounts less than 300 mg may also be justified provided the formulation completely covers the exposed membrane surface and an infinite dose is maintained in the donor chamber at the final sampling point of the experiment. Allowing release of more than $30 \%$ of the applied dose can lead to non-linearity of the release profile as the amount of drug substance in the donor chamber becomes rate limiting. The slope of the release profile will decline leading to the appearance of a saturation of release. Insufficient linearity can contribute to variability in release rate data and impact the overall quality of data; thus, the $30 \%$ release cut-off is recommended to ensure precise and reproducible data.

\section{Application Method}

Because there are multiple acceptable designs of the VDC, the approach for delivering the dosage form into the donor chamber may vary. In addition, the physical properties (e.g., viscosity) of the dosage form itself may be a factor in how best to deliver the drug product into the donor chamber. Various methods have been implemented including using a metal spatula to spread the dosage form into the middle of a Teflon wafer, using syringes of various volumes, and pouring the product directly on the exposed surface of the membrane. The 
approach should minimally affect the rheology of the dosage form and be reproducible, such that release rates are not affected by the application method.

\section{COMMON SOURCES OF VARIABILITY IN IVRT METHODS USING VDC}

As with any analytical methodology, IVRT methods and the calculated release rates have a certain degree of variability. Specific aspects of IVRT methods require significant human intervention (e.g., sample preparation, application of the product to the donor chamber, sampling), which contributes to a higher degree of variability than other well-established analytical methods. Much published literature has described the associated variability, including the original SUPAC guidance document, highlighting the importance of a fundamental understanding of where this variability comes from, and how it might be better controlled $(25-27,10)$. There are several outputs from an IVRT experiment in which variability is frequently observed; understanding how IVRT data is expressed is key to understanding and identifying the sources of potential error. Generally speaking, there are three different categories of variability that can be observed from an IVRT method, referred to as 'withincell variability', 'within-experiment variability', and 'dayto-day variability'.

Release rate data is generated based on linear regression of the cumulative release of the active ingredient per unit area expressed as a function of the square root of time (i.e. the linearity of release rate) (Figure 1); therefore, a release rate is calculated based on a minimum of five samples over a given time period within a single cell. Variability among sample concentrations within a single cell can lead to poor linearity, which can be described as 'within-cell variability' because it occurs within a single diffusion cell. The linearity of the release rate should be $>0.90$ within a single cell to minimize variability amongst multiple cells (12).

Typical experimental protocols include six replicates for a given drug product to allow calculation of an average release rate. Within these six replicates, the coefficient of variation should ideally be $\leq 15 \%$ to allow meaningful conclusions to be drawn; although variability can reach as high as $20 \%$ and still be considered sufficiently precise based on the experience of the authors (12). This type of variability can be described as 'within-experiment variability' because it is calculated from the release rates from multiple diffusion cells within a single experiment.

Release rate experiments for a single product may be performed on multiple days and by multiple scientists. Poorly robust methods can express variability over time between experiments; this type of variability can be described as 'day-to-day variability'. An example of release rate experiments being performed on multiple days for a single product is during a stability program, where release rate data from an initial data point $(T=0)$ may be compared to later data points (e.g., $\mathrm{T}=3$ months) generated after extended periods of time at specified storage conditions. To allow interpretation of data over extended periods of time, VDC methods must be reproducible when performed on different days and by different scientists.

To consistently minimize variability and generate precise and reproducible data, one must consider the contributing factors from the experimental protocol and identify those than can be minimized by improving technique and implementing laboratory controls. Within a given experiment, there are many potential sources of variability including the dosage form itself, appropriate selection of a compatible VDC design, sample preparation, sample application method, sampling technique, and composition of the receptor fluid. Each of these factors will be discussed in greater detail.

\section{Drug Product Dosage Form and Physical Properties}

Certain dosage forms may be more prone to variability than others by nature of their physical properties. Physical properties can affect the rate of release of a drug from the dosage form matrix and contribute to the variability associated with the developed IVRT method. The type of dosage and the complexity of the dosage form will contribute to the extent of variability observed in the release rate profile. Dosage forms with multiple phases (e.g., creams and lotions) are more complex than single-phase dosage forms (e.g., gels); the complex nature of the dosage form makes it more difficult to control the variability. The active ingredient may be required to diffuse through multiple phases within the formulation before being fully released from the dosage form to enter the receptor chamber and receptor fluid. Further, multiplephase dosage forms are frequently more sensitive to shear stress, and their physical stability may be impacted during sample preparation or during application of the sample to the donor chamber.

Similarly, dosage forms that contain a percentage of the drug in solution and a percentage in suspension are another example of more complex dosage forms with a tendency towards increased inherent variability. The active ingredient may require solubilization within the 
dosage form or at the interface with receptor fluid before being fully released into the receptor fluid.

As an example, internal studies have shown that a polar drug, such as acyclovir, often shows high variability in the release profile when present in suspension in ointment, while variability from the cream formulation may be easier to control. Similarly, for drugs such as desonide, where multiple dosage forms (lotion, cream, and ointment) exist, inherent variability of the release profile will vary for each dosage form depending on the complexity of the formulation.

\section{VDC Donor Chamber}

USP Chapter <1724> describes two different donor chamber designs. The first model uses a Teflon wafer to create the donor chamber that will hold the product on top of the synthetic membrane. The wafer is typically $1.5 \mathrm{~mm}$ in height and $15 \mathrm{~mm}$ in diameter, although other dimensions are commercially available. A support disk is assembled on top of the wafer to occlude the product before applying a clamp to secure the donor chamber to the receptor chamber. In this model, the wafer defines the surface area in which the product contacts the membrane, and the product is applied to the donor chamber before fully securing the wafer and disk to the receptor chamber.

The second model uses a ground glass donor chamber in combination with a ground glass receptor chamber to create the interface between the two chambers. The orifice in both chambers has matching diameters, typically 11.28 or $15 \mathrm{~mm}$, thus defining the surface area in which the product contacts the membrane and receptor fluid. In this model, the product is applied to the donor chamber after fully securing the donor chamber to the receptor chamber. The donor chambers in this model are typically $18 \mathrm{~mm}$ height, approximately 10 times greater than the standard wafers.

There are three important differences to note between the two types of donor chambers. Because the Teflon wafer model does not have a corresponding surface underneath the membrane to provide support for the formulation, the wafer model may not be ideal for low viscosity or runny formulations. Using this model with a low-viscosity formulation can lead to the formulation leaking outside of the defined surface area created by the wafer orifice, creating variability in the exposure of the drug product to the receptor fluid, potentially causing 'within-cell variability.' If the surface area of exposure is variable from one diffusion cell to the next, the variability in release rates may also go up within an experiment. The ground glass model is amenable to lowviscosity formulations and is even compatible with liquid formulations as the ground glass joint provides a sufficient interface to prevent leakage.

Secondly, the capacity of the donor chamber when using a Teflon wafer is limited due to reduced height. The capacity of the donor chamber when using the standard size wafer is approximately 200-400 mg of drug product, while the capacity of the donor chamber without the wafer is five to 10 times larger.

The final difference to note is the point at which the donor chamber is fully secured to the receptor chamber. When using the ground glass model, the chambers are secured together before applying the product to the diffusion cell, reducing access to the membrane surface and making it more challenging to gently deliver the formulation to the membrane surface. Due to the reduced access, the sample application methods are limited with this model. For some formulations, the sample application method can alter the physical characteristics of the product and lead to both within-cell and within-experiment variability.

\section{Sample Preparation and Application Method}

The sample preparation technique and application method can lead to substantial variability if not performed properly. Sample preparation refers to the method used to collect the formulation from the package (i.e., tube, pump, jar), and the application method refers to the how the formulation is applied to the donor chamber. The approach to collect sufficient formulation from the dosage form should be simple, consistent, and apply minimal stress or shear to the drug product. Shear stress can create physical instability in complex dosage forms such as lotions and creams; the resulting changes within the formulation can lead to within-cell variability as well as within-experiment variability, as the formulation transforms within the donor chamber following application. In some cases, subtle manipulation or mixing of the formulation prior to application may be necessary, but should be appropriately justified.

Similarly, the application technique should be simple, consistent, and apply minimal stress or shear to the drug product. There are two typical approaches: spreading with a spatula and delivery with a syringe. The approach used is typically dependent on two factors: the type of diffusion cell donor chamber and the physical properties of the dosage form. As described earlier, certain VDCs require the use of a Teflon wafer and others do not. Models that use a Teflon wafer allow greater access to

|Dissolution 
the membrane surface during application, which allows the formulation to be spread gently with a metal spatula, reducing variability for complex formulations that are sensitive to shear stress applied to the formulation if delivered to the donor chamber using a syringe. Therefore, release rates from complex formulations will frequently have reduced variability when the formulation is applied to the donor chamber using a spreading technique with a spatula. As an example, Table 1 presents a comparison of release rate variability using two different application techniques for single-phase and multiple-phase formulations (28). Precision of the release rates was dependent on the application technique for the two-phase products. Minimizing shear during application to the donor chamber led to improved precision and reproducibility of release rates. The impact of shear on the variability was dosage form dependent, as the more complex formulations were impacted while the singlephase product was not. Additionally, Figure 2 presents release rate data for hydrocortisone from a $1 \%$ cream formulation using each application technique. When the product was applied using a spatula, the release rates showed less variability compared to application of the product with a syringe.

The formulation should be applied smoothly and evenly across the membrane and should not visibly separate from the membrane after application. Separation will create air bubbles to form at the interface between the membrane and the drug product. The impact of gaps in contact can have a similar effect on the data as an air bubble in the receptor fluid. The variability can be expressed as within-cell or within-experiment variability. Some products, such as hydrophobic ointments, have an increased tendency to form air pockets during spreading compared to delivery with a syringe. The physical compatibility of the formulation with the membrane will have significant influence on the formation of gaps in the drug product.

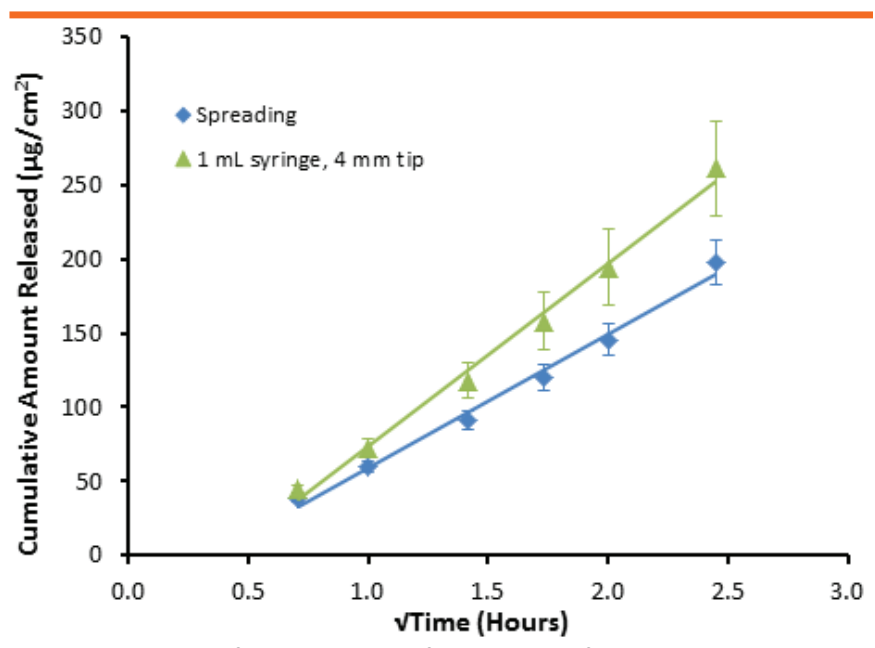

Figure 2. Release of hydrocortisone from a cream formulation using different application techniques.

\section{Sampling Technique}

Sampling technique is a critical component of the method and can contribute to variability in the data if not performed properly. Proper sampling technique differs depending on the manufacturer of the diffusion cell; general suggestions are described in USP <1724>. The sample should be consistently withdrawn from the center of the diffusion cell below the membrane surface. Care should be implemented to ensure the sample is not taken in close proximity to the arm of the diffusion cell, as mixing in the base of the arm is minimal in comparison to the center of the diffusion cell. Sampling too close to the arm of the diffusion cell can lead to low sample concentrations and apparent non-linearity in release rate of the active ingredient. If the sampling procedure is not performed using a calibrated pipette, the procedure should be appropriately qualified to demonstrate that it does not contribute to within-cell or within-experiment variability.

Table 1. Release Rate Variability for Three Drug Products Using Two Distinct Application Techniques $(n=6)$

\begin{tabular}{|c|c|c|c|c|c|c|c|c|c|c|c|}
\hline Drug Product & $\begin{array}{l}\text { Application } \\
\text { Technique }\end{array}$ & \multicolumn{5}{|c|}{$\begin{array}{c}\text { Syringe } \\
\text { (higher shear) }\end{array}$} & \multicolumn{5}{|c|}{$\begin{array}{l}\text { Spatula } \\
\text { (minimal shear) }\end{array}$} \\
\hline \multirow{2}{*}{ Two-Phase Cream } & Exp. \# & 1 & 2 & 3 & - & - & 1 & 2 & 3 & 4 & 5 \\
\hline & $\%$ RSD & 24.5 & 30.7 & 25.5 & - & - & 6.5 & 15.5 & 7.8 & 14.1 & $8.7-$ \\
\hline \multirow{2}{*}{ Two-Phase Gel } & Exp.\# & 1 & 2 & 3 & 4 & 5 & 1 & 2 & 3 & 4 & - \\
\hline & $\% \mathrm{RSD}$ & 32.7 & 28.6 & 20.4 & 29.3 & 36.3 & 3.1 & 10.1 & 1.0 & 9.1 & - \\
\hline \multirow{2}{*}{ Single-Phase Ointment } & Exp.\# & 1 & 2 & 3 & - & - & 1 & 2 & 3 & - & - \\
\hline & \%RSD & 12.3 & 5.4 & 6.6 & - & - & 17.1 & 17.1 & 14.1 & - & - \\
\hline
\end{tabular}

*Exp, experiment; RSD, relative standard deviation; -, not tested. 
After sampling or during sampling, it is important to replace the volume sampled with an equivalent volume of pre-warmed receptor fluid to maintain a constant volume during the experiment. During both sampling and replacing it is critical to avoid the introduction of any air bubbles into the receptor chamber. Air bubbles will rise to the interface between the receptor fluid and inert membrane, reducing the surface area available for drug release. This reduced surface area can lead to a reduction in release rate and contribute to within-cell variability.

\section{Receptor Fluid Composition}

The composition of the receptor fluid is arguably the most critical parameter in any IVRT method. The composition of the receptor fluid should be compatible with the drug product and the inert membrane, the active ingredient should be sufficiently soluble in the volume available for the test, and it should facilitate discrimination sensitivity, selectivity and specificity. During development and validation of the method, the sensitivity of the release rate to the composition of the receptor fluid should be assessed during robustness experiments. The ratio of components, $\mathrm{pH}$, ionic strength, and any other relevant variations should be evaluated. The release rate of the active ingredient using the altered compositions should be directly compared to the release rate of the active ingredient using the original method conditions. Methods with insufficient robustness (i.e., when the altered conditions do not meet the "sameness" criteria using the statistical test described in the SUPAC guidance (10)) can be poorly reproducible and lead to high inter-experiment variability between different days, labs, or scientists (i.e., day-to-day variability). Figure 3 illustrates an example in which the release rate of the active ingredient is highly sensitive to the amount of organic solvent present in the receptor fluid. Moderate adjustments to the amount of organic solvent ( $5 \%$ ) led to substantial differences in the release rate of the active ingredient. In this case, adding a small amount of additional organic solvent to the receptor fluid doubled the release rate. Sensitivity such as this will make it difficult to compare release rates generated on different days and by different scientists using different receptor fluid preparations. Highly controlled techniques and tight limits for preparation of the receptor fluid can minimize the impact of a highly sensitive receptor fluid (i.e., the use of volumetric glassware and preparation of the receptor fluid on the day of use).

\section{Receptor Fluid Outgassing (Air Bubbles)}

A very common source of variability is caused by receptor fluid outgassing and formation of air bubbles in the receptor chamber. Receptor fluid outgassing is prevalent in receptor fluids that contain mixtures of aqueous and organic components. Receptor fluids with surfactants also tend to form air bubbles during an IVRT experiment and are typically used only when necessary to achieve adequate solubility of the active ingredient. These air bubbles can collect at the interface between the membrane and the receptor fluid, reducing the surface area available for drug release. This reduced surface area can lead to a reduction in release rate and contribute to variability in the data. If air bubbles are observed during an experiment, they can be removed by carefully inverting the cell to release the bubble through the open sampling arm. Depending on when an air bubble is identified, the variability may be expressed as 'withincell' as non-linearity, or 'within-experiment' as an outlier cell. De-aeration of the receptor fluid prior to use will limit outgassing during the experiment.

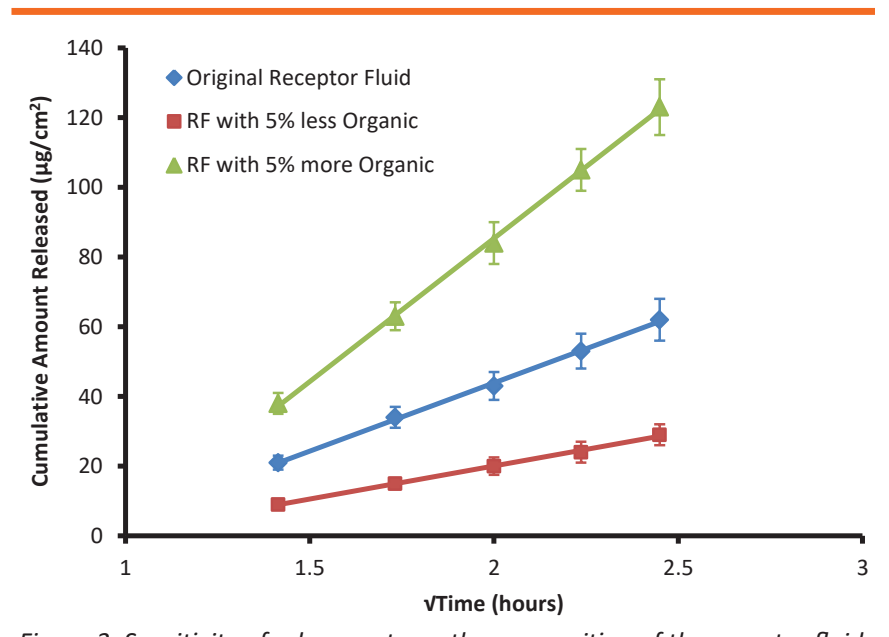

Figure 3. Sensitivity of release rate on the composition of the receptor fluid.

\section{CONCLUSIONS}

There are several factors that contribute to the overall variability observed in IVRT data. Current equipment used to perform IVRT studies requires considerable human involvement, particularly during sample application and sample withdrawal, making data vulnerable to experimental error. Implementation of strict laboratory controls and experimental protocols, as well as using appropriately qualified and validated VDC systems, can reduce the likelihood of higher variability. As IVRT methodology continues to evolve into a regulatory expectation, standardized equipment and enhanced automation will continue to reduce the observed variability and improve experimental outcomes.

\section{ACKNOWLEDGEMENTS}

The authors disclosed no funding related to this article. 


\section{CONFLICT OF INTEREST}

The authors disclosed no conflicts of interest related to this article.

\section{REFERENCES}

1. Zatz,J.L.;Varsano, J.;Shah, V.P. In vitro release of betamethasone dipropionate from petrolatum-based ointments. Pharm. Dev. Technol. 1996, 1, 293-298. DOI: 10.3109/10837459609022598.

2. Shah, V. P.; Elkins, J.; Hanus, J.; Noorizadeh, C.; Skelly, J. P. In vitro release of hydrocortisone from topical preparations and automated procedure. Pharm. Res. 1991, 8, 55-59. DOI: 10.1023/A:1015826205930.

3. Franz, T. J. On the diffusion of tritiated water through skin. J. Invest. Dermatol. 1968, 50, 260. DOI: 10.1038/jid.1968.38.

4. $\quad<1724>$ Semisolid Drug Products - Performance Tests. In United States Pharmacopeia and National Formulary USP 41-NF 36; United States Pharmacopeial Convention, Inc.; Rockville, MD, 2018, pp 7944-7956.

5. Flynn, G. L.; Shah, V. P.; Tenjarla, S. N.; Corbo, M.; DeMagistris, D.; Feldman, T. G.; Franz, T. J.; Miran, D. R.; Pearce, D. M.; Sequeira, J. A.; Swarbrick, J.; Wang, J. C.; Yacobi, A.; Zatz, J. L. Assessment of value and applications of in vitro testing of topical dermatological Products Pharm. Res. 1999, 16, 13251330. DOI: 10.1023/A:1018997520950.

6. Olejnik, A.; Goscianska, J.; Nowak, I. Active compounds release from semisolid dosage forms. J. Pharm. Sci. 2012, 101, 40324045. DOI: 10.1002/jps.23289.

7. Shah, V. P.; Yacobi, A.; Radulescu, F. S.; Miron, D. S.; Lane, M. E. A science based approach to topical drug classification system. Int. J. Pharm. 2015, 491, 21-25. DOI: 10.1016/j. ijpharm.2015.06.011.

8. Shah, V. P.; Radulescu, F. S.; Miron, D. S.; Yacobi, A. Commonality between BCS and TCS. Int. J. Pharm. 2016, 509, 35-40. DOI: 10.1016/j.ijpharm.2016.05.032.

9. Kanfer, I.; Rath, S.; Purazi, P.; Mudyahoto, N. A. In vitro release testing of semi-solid dosage forms. Dissol. Technol. 2017, 24, 52-60. DOI: 10.14227/DT240317P52.

10. Nonsterile Semisolid Dosage Forms. Scale-Up and Postapproval Changes: Chemistry, Manufacturing, and Controls; In Vitro Release Testing and In Vivo Bioequivalence Documentation. Guidance for Industry; U.S. Department of Health and Human Services, Food and Drug Administration, Center for Drug Evaluation and Research (CDER), U.S. Government Printing Office: Washington, DC, 1997.

11. Draft Guidance on Acyclovir. U.S. Department of Health and Human Services, Food and Drug Administration, Office of Generic Drugs, March 2012.

12. Draft Guidance on Acyclovir. U.S. Department of Health and Human Services, Food and Drug Administration, Office of Generic Drugs, December 2016.

13. Draft Guidance on Dapsone. U.S. Department of Health and
Human Services, Food and Drug Administration, Office of Generic Drugs, October 2017.

14. Draft Guidance on Ivermectin. U.S. Department of Health and Human Services, Food and Drug Administration, Office of Generic Drugs. October 2017.

15. Parera Morell, J. L.; Contreras Claramonte, M. D.; Parera Vialard, A. Validation of a release diffusion cell for topical dosage forms. Int. J. Pharm. 1996, 137, 49-55. DOI: 10.1016/03785173(95)04421-3.

16. Thakker, K. D.; Chern, W. H. Development and validation of in vitro release tests for semisolid dosage forms - case study. Dissol. Technol. 2003, 10, 10-15. DOI: 10.14227/DT100203P10.

17. Ng, S. F.; Rouse, J. J.; Sanderson, F. D.; Meidan, V.; Eccleston, G. M. Validation of a static Franz diffusion cell system for in vitro permeation studies. AAPS PharmSciTech 2010, 11, 1432-1441. DOI: 10.1208/s12249-010-9522-9.

18. Tiffner, K. I.; Kanfer, I.; Augustin, T.; Raml, R.; Raney, S. G.; Sinner, F. A comprehensive approach to qualify and validate the essential parameters of an in vitro release (IVRT) method for acyclovir cream, 5. Int. J. Pharm. 2018, 535, 217-227. DOI: 10.1016/j.ijpharm.2017.09.049.

19. Bioanalytical Method Validation. Guidance for Industry; U.S. Department of Health and Human Services, Food and Drug Administration, Center for Drug Evaluation and Research (CDER), Center for Veterinary Medicine (CVM), May 2018.

20. International Conference on Harmonisation of Technical Requirements for Registration of Pharmaceuticals for Human Use. Validation of Analytical Procedures: Text and Methodology, Q2(R1); ICH Harmonised Tripartite Guideline: Geneva, Switzerland, 2005. http://www.ich.org/fileadmin/ Public_Web_Site/ICH_Products/Guidelines/Quality/Q2_R1/ Step4/Q2_R1__Guideline.pdf (accessed July 18, 2018).

21. Higuchi, T. Rate of release of medicaments from ointment bases containing drugs in suspension. J. Pharm. Sci. 1961, 50, 861865. DOI: $10.1002 / j p s .2600501018$.

22. Higuchi, T. Analysis of data on the medicament release from ointments. J. Pharm. Sci. 1962, 51, 802-804. DOI: 10.1002/ jps. 2600510825.

23. Higuchi, T. Mechanism of sustained action medication. Theoretical analysis of release of solid drugs dispersed in solid matrices. J. Pharm. Sci. 1963, 52, 1145-1149. DOI: 10.1002/ jps.2600521210.

24. Siepmann, J.; Peppas, N. A. Higuchi equation: derivation, application, use and misuse. Int. J. Pharm. 2011, 418, 6-12. DOI: 10.1016/j.ijpharm.2011.03.051.

25. Hauck, W. H.; Shah, V. P.; Shaw, S. W.; Ueda, C. T. Reliability and reproducibility of vertical diffusion cells for determining release rates from semisolid dosage forms. Pharm. Res. 2007, 24, 20182024. DOI: 10.1007/s11095-007-9329-x.

26. Klein, S. Influence of different test parameters on in vitro drug release from topical diclofenac formulations in a vertical 
diffusion cell setup. Pharmazie 2013, 68, 565-571. DOI: 10.1691/ph.2013.6528.

27. Petro, E.; Paal, T. L.; Eros, I.; Kenneth, A. S.; Baki, G.; Csoka, I. Drug release from semisolid dosage forms: a comparison of two testing methods. Pharm. Dev. Technol. 2015, 20, 330-336. DOI: 10.3109/10837450.2013.867446.

28. Klein, R. R.; Reynolds, J.; Vinjamuri, B. P.; Voss, P.; Thorpe, T.; McClellan, M. B.; Stewart, B.; Thakker, K. D. In vitro release of actives from semi-solid formulations is highly dependent on application technique and linked to altered microstructure from shear during application. Poster 24T0930; 2016 AAPS Annual Meeting and Exposition, Denver, CO, November 13-17, 2016.

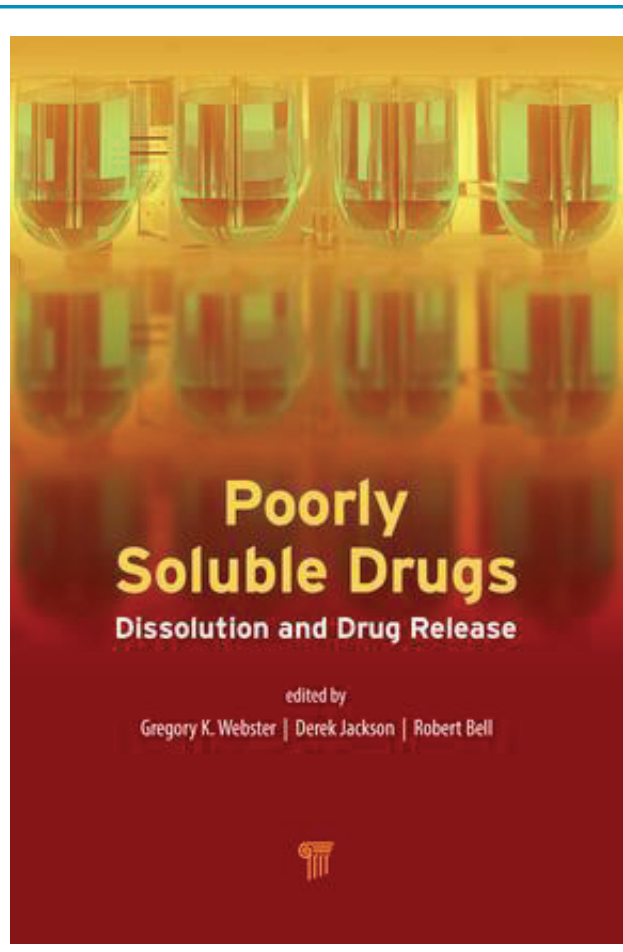

\section{Poorly Soluble Drugs}

Dissolution and Drug Release

By Gregory K. Webster, Robert G. Bell, J. Derek Jackson

(C) 2016 - Pan Stanford

728 pages | 149 B/W IIlus.

List: $\$ 249.95$ (US), $\$ \mathbf{2 7 5 . 0 0}$ (Overseas) Order online at www.dissolutiontech.com, price includes shipping and handling Hardback: 9789814745451 pub: 2016-12-20

\section{About the Book}

This book is the first text to provide a comprehensive assessment of the application of fundamental principles of dissolution and drug release testing to poorly soluble compounds and formulations. Such drug products are, vis-à-vis their physical and chemical properties, inherently incompatible with aqueous dissolution. However, dissolution methods are required for product development and selection, as well as for the fulfillment of regulatory obligations with respect to biopharmaceutical assessment and product quality understanding. The percentage of poorly soluble drugs, defined in classes 2 and 4 of the Biopharmaceutics Classification System (BCS), has significantly increased in the modern pharmaceutical development pipeline. This book provides a thorough exposition of general method development strategies for such drugs, including instrumentation and media selection, the use of compendial and non-compendial techniques in product development, and phase-appropriate approaches to dissolution development. 